\title{
Liver sinusoidal endothelial cell progenitor cells promote liver regeneration in rats
}

\author{
Lin Wang, ${ }^{1}$ Xiangdong Wang, ${ }^{1}$ Guanhua Xie, ${ }^{1}$ Lei Wang, ${ }^{1}$ Colin K. Hill, ${ }^{2}$ and Laurie D. DeLeve ${ }^{1}$
}

1 Division of Gastrointestinal and Liver Disease and University of Southern California Research Center for Liver Disease and
2Department of Radiation Oncology, Keck School of Medicine, University of Southern California, Los Angeles, California, USA

\begin{abstract}
The ability of the liver to regenerate is crucial to protect liver function after injury and during chronic disease. Increases in hepatocyte growth factor (HGF) in liver sinusoidal endothelial cells (LSECs) are thought to drive liver regeneration. However, in contrast to endothelial progenitor cells, mature LSECs express little HGF. Therefore, we sought to establish in rats whether liver injury causes BM LSEC progenitor cells to engraft in the liver and provide increased levels of $\mathrm{HGF}$ and to examine the relative contribution of resident and $\mathrm{BM}$ LSEC progenitors. LSEC label-retaining cells and progenitors were identified in liver and LSEC progenitors in BM. BM LSEC progenitors did not contribute to normal LSEC turnover in the liver. However, after partial hepatectomy, BM LSEC progenitor proliferation and mobilization to the circulation doubled. In the liver, onequarter of the LSECs were BM derived, and BM LSEC progenitors differentiated into fenestrated LSECs. When irradiated rats underwent partial hepatectomy, liver regeneration was compromised, but infusion of LSEC progenitors rescued the defect. Further analysis revealed that BM LSEC progenitors expressed substantially more HGF and were more proliferative than resident LSEC progenitors after partial hepatectomy. Resident LSEC progenitors within their niche may play a smaller role in recovery from partial hepatectomy than BM LSEC progenitors, but, when infused after injury, these progenitors engrafted and expanded markedly over a 2-month period. In conclusion, LSEC progenitor cells are present in liver and BM, and recruitment of BM LSEC progenitors is necessary for normal liver regeneration.
\end{abstract}

\section{Introduction}

The liver is the only human internal solid organ capable of regeneration after resection. The current literature suggests that normal liver regeneration requires increased expression of hepatocyte growth factor (HGF) by liver sinusoidal endothelial cells (LSECs) (1-3) and LSEC proliferation (4-6); however, mature LSECs, like other endothelial cells, are low in $\operatorname{HGF}(2,7)$, whereas endothelial progenitor cells (EPCs) are both rich in HGF (7) and highly proliferative. This raises the question as to whether the increase in HGF indeed occurs in mature LSECs or whether after injury there is an influx of LSEC progenitor cells (SPCs) that proliferate and provide the increase in HGF.

The LSEC is unique in its morphology and function (8). One difference from other endothelial cells is that more than $80 \%$ of LSECs, i.e., LSECs in the periportal and midlobular regions of the liver, express CD45 on the cell surface $(8,9)$ and by gene expression (8). CD45 expression by LSECs indicates that they are not derived from the CD45- EPC that has been described as the progenitor to other endothelial cells.

In addition to BM-derived EPCs, recent studies have suggested the existence of resident EPCs in the vasculature. This evidence, however, is predominantly based on in vitro cell culture assays and phenotypic marker studies $(10,11)$. Important questions have been raised about what role the resident EPC would play in turnover of endothelial cells, whether it is transiently present, whether it derives from a BM stem cell, and whether a resident stem cell can be identified (10). Given the availability of methods to isolate LSECs with very high purity and in large numbers, the liver is an ideal organ to examine the existence of resident label-retaining cells (LRCs) and resident SPCs.

Conflict of interest: The authors have declared that no conflict of interest exists. Citation for this article: J Clin Invest. 2012;122(4):1567-1573. doi:10.1172/JCI58789.
This study has identified 2 distinct populations of LSEC progenitors: LSEC LRCs and SPCs that reside within the liver and SPCs present in the BM. The study examines the BM progenitor cell response to partial hepatectomy $(\mathrm{PHx})$, persistence of SPC engraftment, and magnitude of SPC expansion in the liver as well as the requirement for SPC recruitment in liver regeneration and the relative contribution of resident and BM SPCs to repair of liver injury.

\section{Results}

Identification of resident LSEC LRCs and progenitors. The LSEC expresses both CD31 and CD45 $(8,9)$. CD133 is a progenitor cell marker, and infusion of $\mathrm{CD}_{133^{+}}$cells from the BM repopulates the liver endothelium (8). We therefore used $\mathrm{CD} 133^{+} \mathrm{CD} 45^{+} \mathrm{CD} 31^{+}$triple positivity as the phenotypic signature for SPCs.

To identify resident SPCs, a nonparenchymal cell population was subjected to iodixanol density gradient centrifugation $(12,13)$, and elutriation fractions were examined for $\mathrm{CD}_{133^{+}}$cells. $\mathrm{CD}_{133^{+}}$cells were only present in the elutriation fraction that contains LSECs, indicating that CD $133^{+}$LSECs are the same size as mature LSECs. $90.9 \% \pm 2.3 \%$ of CD $133^{+}$LSECs were CD $45^{+}$ and $93.1 \% \pm 6.0 \%$ of CD $133^{+}$LSECs were CD $31^{+}$(Figure 1, A-C); the percentages were similar in the CD133-depleted fraction of LSECs, $91.8 \% \pm 7.7 \%$ and $90.7 \% \pm 1.7 \%$, respectively $(n=3)$. The resident $\mathrm{CD} 133^{+} \mathrm{CD} 45^{+} \mathrm{CD} 31^{+} \mathrm{SPCs}$ isolated by this method showed the hallmark ultrastructural features of LSECs, i.e., fenestrae organized in sieve plates, as identified by scanning EM (Figure 1D). Thus, these CD $133^{+}$cells were resident SPCs, with ultrastructural features that identify them as LSECs. One hundred percent of resident SPCs stained positive for the endothelial markers VEGF-receptor 1 and VEGF-receptor 1 (Figure 2). The pattern of VEGF receptor staining was identical to the staining pattern of mature LSECs, periportal LSECs (CD45 bright LSECs; 

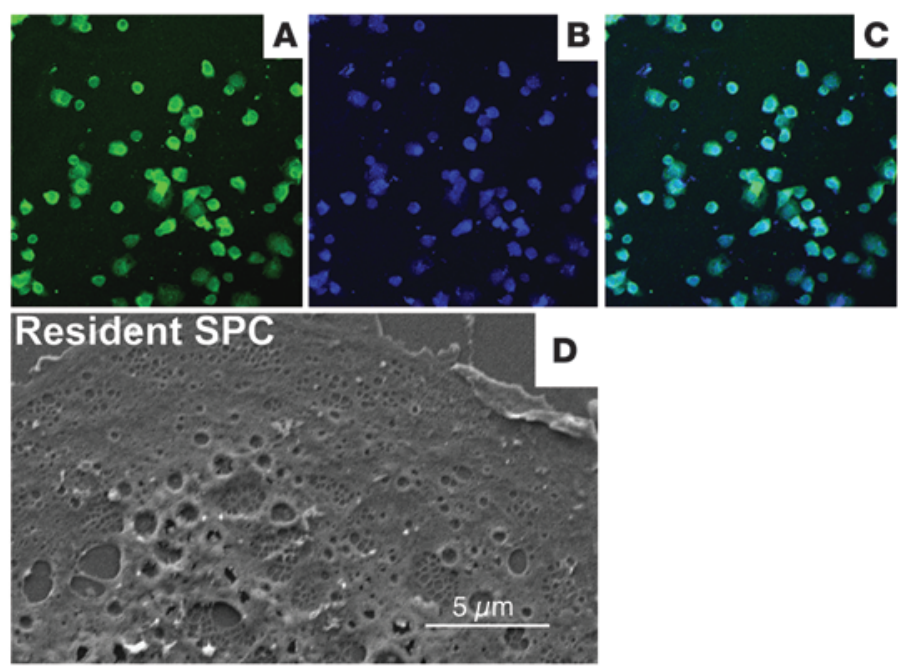

ref. 14), and centrilobular LSECs (CD45- LSECs; ref. 14 and Figure 2). The frequency of resident SPCs varied across strains of rats: $1.1 \% \pm 0.1 \%$ of LSECs in Sprague-Dawley rats were CD $133^{+}$, $1.5 \% \pm 0.1 \%$ of LSECs in Lewis rats were CD $133^{+}$, and $7.0 \% \pm 0.3 \%$ of LSECs in Fischer rats were $\mathrm{CD}_{133^{+}}(n=3)$. As described below, infusion of resident SPCs during liver injury leads to persistent engraftment and expansion as LSECs.

\section{Figure 1}

Resident SPCs. LSECs were isolated by elutriation, and the CD133+ fraction was obtained by immunomagnetic separation to obtain resident SPCs. (A) Staining for CD45 (FITC; green). (B) Staining for CD31 (Alexa Fluor 405; blue). (C) The merged image of $\mathbf{A}$ and $\mathbf{B}$ demonstrates that these $\mathrm{CD} 133^{+}$cells are also CD $45^{+}$CD $31^{+}$. Original magnification, $\times 100$. (D) Scanning EM demonstrates the resident SPCs have fenestration organized in sieve plates characteristic of LSECs. Scale bar: $5 \mu \mathrm{m}$.
To determine whether there was a population of label-retaining LSECs within the liver, BrdU was injected at birth, and cells were isolated at 2 months after birth $(n=3)$. As expected, none of the $\mathrm{BrdU}^{+}$cells were present in the $\mathrm{CD} 133^{-}$fraction. The $\mathrm{BrdU}^{+}$ cells obtained by immunomagnetic selection for CD133 from the LSEC elutriation fraction were $\mathrm{CD} 133^{+} \mathrm{CD} 45^{+} \mathrm{CD} 31^{+}$(Figure 3), indicating that the $\mathrm{BrdU}^{+}$cells obtained by this method were label-

\section{Figure 2}

VEGF receptor staining. Resident SPCs (CD133+ fraction of LSECs), LSECs with removal of resident SPCs (fraction of LSECs depleted of $\mathrm{CD} 133^{+}$cells), periportal LSECs (CD45 bright), and centrilobular LSECs (CD45 LSECs) were stained for VEGF-receptor 1 (VEGF-R1) and VEGF-R2. The third column shows a merged image. Original magnification, $\times 100$.

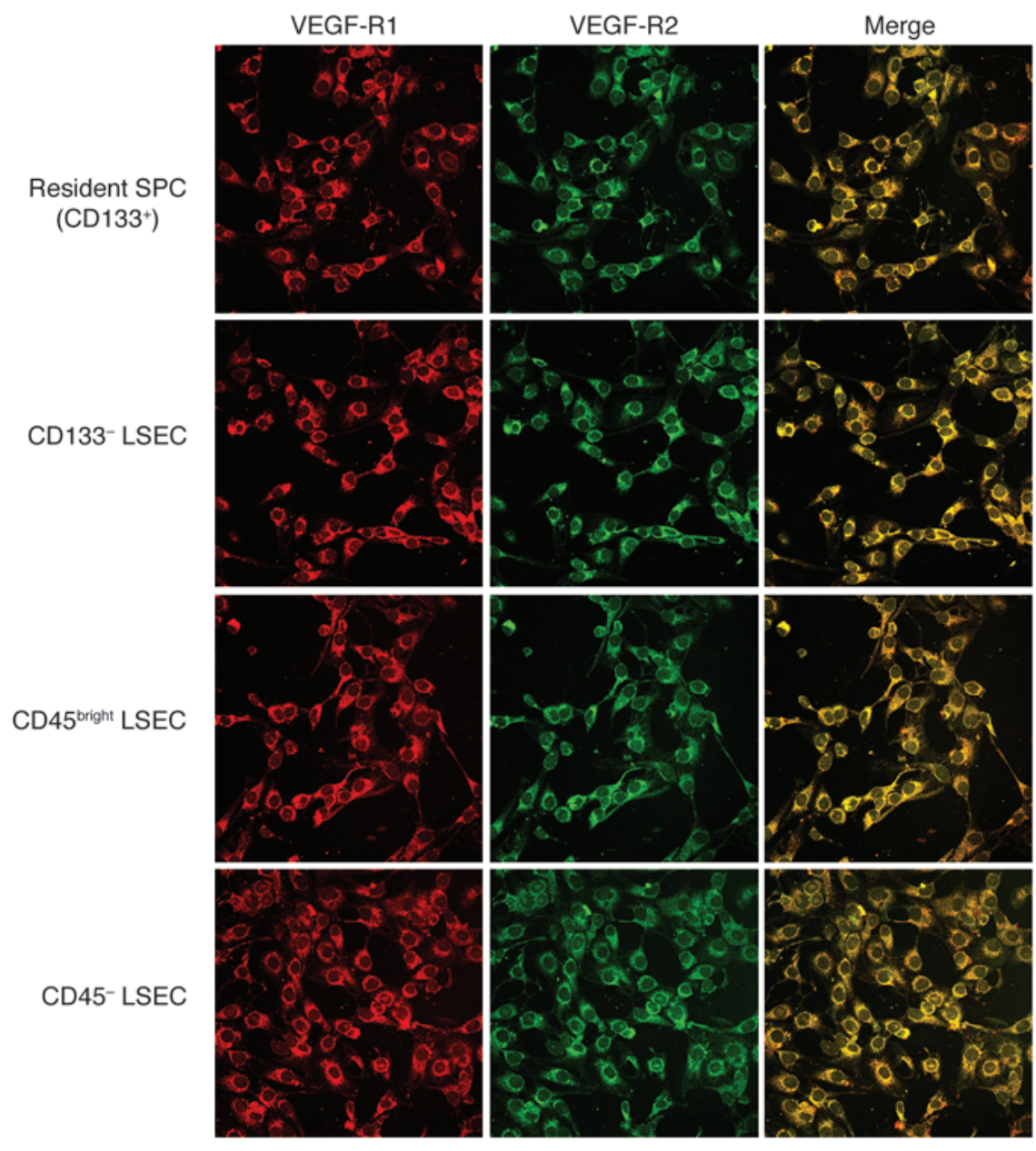



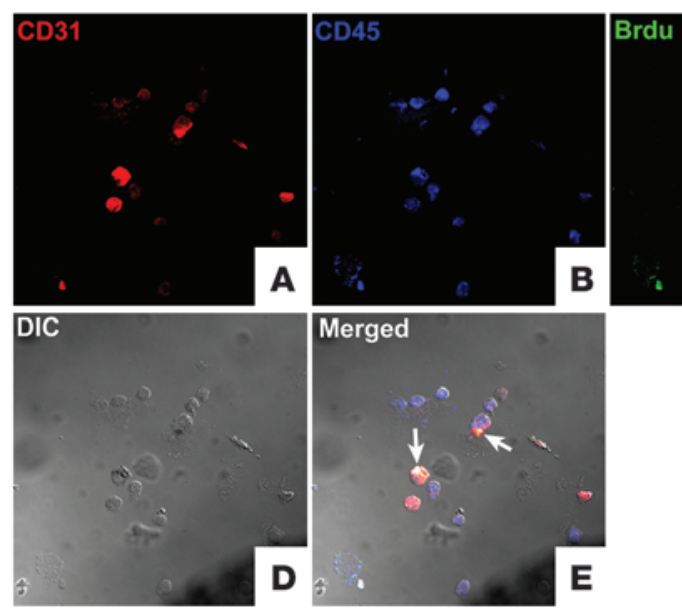

Merged
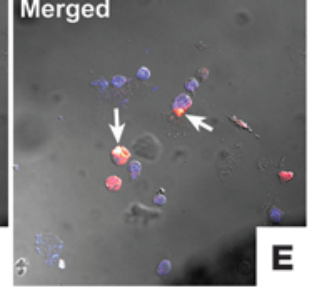

retaining LSEC progenitors. $1.1 \%$ of LSECs in this rat strain were $\mathrm{CD}_{133^{+}}$, and $9.1 \% \pm 2.3 \%$ of the $\mathrm{CD} 133^{+}$cells were $\mathrm{BrdU}{ }^{+}$, so that $0.1 \%$ of the LSEC population was resident LSEC LRCs.

BM SPCs and normal LSEC turnover. To investigate the role of BM SPCs in normal LSEC turnover, gender-mismatched BM transplantation (male into female) was performed, and LSECs were isolated 9 months later. LSECs derived from the BM were identified in recipient rats by FISH detection of the Y chromosome (8). Only $0.8 \% \pm 0.1 \%$ of LSECs and $2.7 \% \pm 0.2 \%$ of resident SPCs were of BM origin at 9 months, demonstrating that BM SPCs play an insignificant role in normal LSEC turnover and therefore do not contribute significantly in uninjured liver to the population of resident SPCs in adult rats.

Peak of LSEC proliferation. To investigate the time course of LSEC proliferation during liver regeneration, LSECs were isolated after $\mathrm{PHx}$ and immunostained for proliferating cell nuclear antigen (PCNA) (Figure 4A). Fewer than 2\% of LSECs were $\mathrm{PCNA}^{+}$after sham surgery. LSEC proliferation reached a peak on day 3 , with nearly $40 \%$ PCNA $^{+}$LSECs.

Progenitor cell response. If BM SPCs contribute to liver regeneration, then PHx should elicit a progenitor cell response, defined here as proliferation of SPCs in the BM, mobilization of SPCs from BM to the circulation, engraftment of SPCs in the liver, and differentiation of SPCs into LSECs.

SPCs $\left(\mathrm{CD} 133^{+} \mathrm{CD} 45^{+} \mathrm{CD} 31^{+}\right.$cells) were isolated from the $\mathrm{BM}$ on day 3 after $\mathrm{PHx}$ or after sham surgery. After $\mathrm{PHx}$, proliferation of SPCs within the BM increased from $20 \%$ in the sham-operated group to $50 \%$ in the PHx group (Figure 4B). The number of BM SPCs mobilized to the circulation after PHx doubled (Figure 4C). The degree of engraftment of BM SPCs was determined with 2 different methods. In gender-mismatched BM-transplanted rats (male BM into female rats), nearly $25 \%$ of isolated LSECs were $\mathrm{Y}$ chromosome positive by FISH on day 3 after $\mathrm{PHx}$, compared with less than $1 \%$ in the sham surgery control group (Figure 4, D-F), demonstrating significant engraftment of BM SPCs after PHx. These findings were confirmed in wild-type Lewis rats transplanted with BM from male Lew-Tg(CAG-EGFP)ys Lewis rats; in these transplanted rats, all BM cells stably expressed GFP. On day 3 after PHx in Lewis rats transplanted with BM from Lew$\operatorname{Tg}$ (CAG-EGFP)ys Lewis rats, nearly $25 \%$ of isolated LSECs were $\mathrm{GFP}^{+}$, i.e., of BM origin (Figure 4G). Figure 5 shows the engraftment of $\mathrm{GFP}^{+} \mathrm{BM}$ SPCs on day 5 after PHx in the livers from wild-

\section{Figure 3}

Resident LRCs. Two months after neonates were injected with BrdU, LSECs were isolated by elutriation, and the $\mathrm{CD}_{133^{+}}$fraction was isolated by immunomagnetic separation to obtain resident SPCs and LRCs. (A) CD31 staining of resident SPCs and LRCs. (B) CD45 staining of resident SPCs and LRCs. (C) BrdU+ staining indicates LRCs (white arrows). (D) Differential interference contrast (DIC) demonstrates all cells in the field. (E) The merged image shows $\mathrm{CD}_{133^{+}}$cells that are CD $31^{+} \mathrm{CD} 45^{+} \mathrm{BrdU}+\mathrm{LRCs}$ (white), indicated by white arrows. Original magnification, $\times 100$. type rats with $\mathrm{BM}$ from Lew-Tg(CAG-EGFP)ys Lewis rats. The presence of $\mathrm{GFP}^{+}$cells (Figure 5A) indicates engrafted BM cells, CD31 staining identifies LSECs (Figure 5B), and colocalization of $\mathrm{GFP}^{+} \mathrm{CD}^{2} 1^{+}$(Figure 5C) cells along the sinusoid demonstrates engraftment of BM SPCs as endothelial cells.

Differentiation of BM SPCs to LSECs. Wild-type Lewis rats transplanted with Lew-Tg(CAG-EGFP)ys BM were used to determine whether BM SPCs that engraft in the liver differentiate into LSECs. LSECs were isolated 2 weeks after $\mathrm{PHx}$, and the $\mathrm{GFP}^{+}$ fraction (i.e., BM-derived cells) was cultured overnight. Scanning EM showed that $\mathrm{GFP}^{+}$LSECs had developed normal fenestrae grouped into sieve plates (Figure $4 \mathrm{H}$ ), the hallmark feature of differentiated LSECs. In contrast, SPCs in the BM were not fenestrated (data not shown).

Persistence of engraftment and expansion. To determine whether engrafted SPCs persist in the liver, resident SPCs were isolated from $\mathrm{GFP}^{+}$transgenic rats, and $1 \times 10^{6}$ resident SPCs were infused into the tail veins of wild-type Lewis rats 3 days after PHx $(n=3)$. Two months later, LSECs were isolated and examined by FACS for GFP expression. $54.2 \% \pm 5.1 \%$ of LSECs and $44.4 \% \pm 3.0 \%$ of resident SPCs were $\mathrm{GFP}^{+}$, demonstrating persistent engraftment of infused resident SPCs (Figure 6). $1 \times 10^{6} \mathrm{GFP}^{+}$resident SPCs were infused, and the total number of $\mathrm{GFP}^{+}$LSECs isolated after 2 months averaged $34.3 \times 10^{6} \pm 5.1 \times 10^{6}$, so that there was at least a 34-fold expansion of infused SPCs to LSECs.

Impairment of liver regeneration by BM suppression and effect of SPC infusion. To examine whether recruitment of BM SPCs is necessary for normal liver regeneration, $\mathrm{PHx}$ was performed in BMsuppressed rats. Rats underwent selective hind limb irradiation, which suppressed the peripheral leukocyte count by nearly $40 \%$ after 1 week. One week after irradiation, rats underwent $\mathrm{PHx}$. Rats received tail vein infusions of either saline ("no infusion"), $1 \times 10^{6}$ resident SPCs, or $50 \times 10^{6} \mathrm{BM}$ cells on day 1 after $\mathrm{PHx}$ and were sacrificed on day 5 (Figure $7 \mathrm{~A})$. One group of rats underwent $\mathrm{PHx}$ without prior irradiation ("PHx only"). Five days after $\mathrm{PHx}$, liver weight of the $\mathrm{PHx}$-only group reached $86 \%$ of that of control littermates (Figure 7B). The amount of liver weight regained in the $B M$-suppressed rats infused with saline (no infusion) was $40 \%$ less than that in PHx-only rats (Figure 7B). Infusion of either resident SPCs or BM cells 1 day after PHx completely reversed the impairment of liver regeneration caused by BM suppression (Figure 7B), whereas infusion on 3 days 


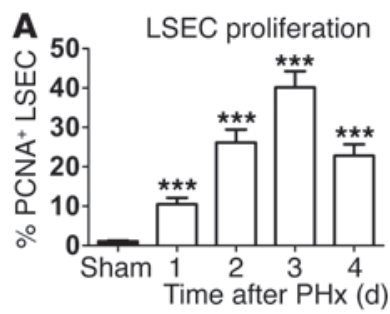

D

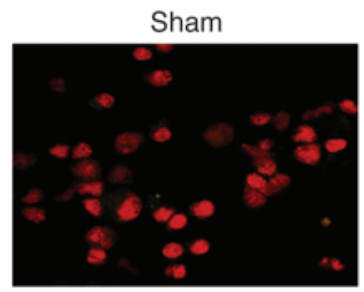

G

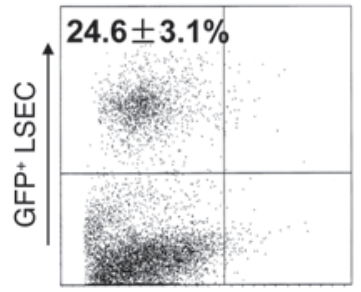

B Proliferation BM SPC

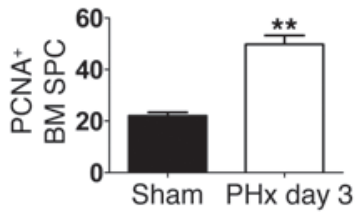

$\mathbf{E}$

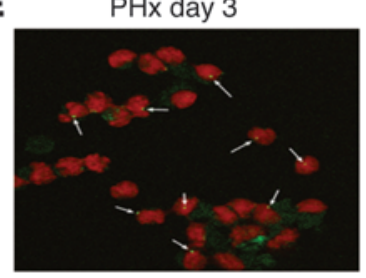

H

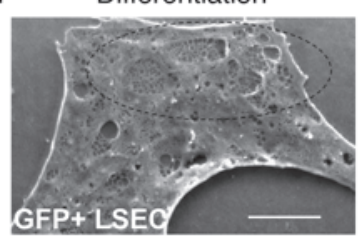

C Mobilization BM SPC

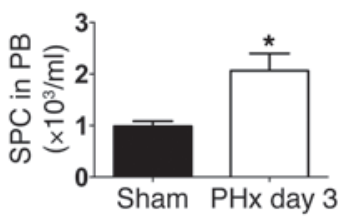

$\mathbf{F}$

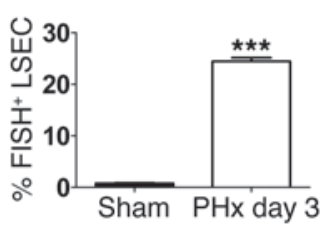

Figure 4

Proliferation, mobilization, engraftment, and differentiation of BM SPCs after PHx. (A) Peak of LSEC proliferation after PHx was examined by PCNA immunostaining in LSECs isolated from sham-operated rats on days 1-4 after PHx. $n=3$; ${ }^{* *} P<0.001$ compared with sham control. (B) Proliferation of BM SPCs. FACS analysis of PCNA+ BM SPCs on PHx day 3 and sham controls. ${ }^{* *} P<0.005$ compared with sham surgery. (C) Mobilization. BM SPCs in peripheral blood (PB) were counted on PHx day 3. $n=4$; ${ }^{*} P<0.05$ compared with sham surgery control. (D-F) Engraftment of BM SPCs. LSECs were isolated on PHx day 3 from rats that had undergone gender-mismatched (male into female) BM transplantation and subsequent $\mathrm{PHx}$. BM-derived LSECs were identified by FISH staining for $\mathrm{Y}$ chromosome (arrows) in (D) sham surgery control and $(E)$ after PHx. Original magnification, $\times 100$. (F) Engraftment data from 3 individual experiments. ${ }^{* \star} P<0.001$ compared with sham surgery control. (G) Engraftment of BM SPCs. Wild-type rats were transplanted with BM from Lew-Tg(CAG-EGFP)ys rats. Isolation of LSECs on PHx day 3, followed by FACS for GFP, demonstrated that $24.6 \% \pm 3.1 \%$ of LSECs were GFP+ and therefore BM derived $(n=3)$. (H) Differentiation of BM SPCs to LSECs. Wild-type rats received BM transplantation from Lew$\mathrm{Tg}(\mathrm{CAG}-\mathrm{EGFP})$ ys rats. BM-derived LSECs $\left(\mathrm{GFP}^{+}\right)$were isolated 2 weeks after $\mathrm{PHx}$ and examined by scanning EM for fenestration. The black circle demonstrates characteristic fenestrae. Original magnification, $\times 5,000$; scale bar: $5 \mu \mathrm{m}$. Note that SPCs isolated from BM were not fenestrated (data not shown).

after PHx did not significantly improve liver regeneration (data not shown). Hepatocyte proliferation was examined by PCNA immunostaining 5 days after $\mathrm{PHx}$ (Figure 7C). The $\mathrm{PCNA}^{+}$fraction of hepatocytes was low in saline-infused, BM-suppressed rats, despite a liver weight that was only $65 \%$ of that of control littermates on day 5 after PHx. However, infusion of resident SPCs or BM cells into BM-suppressed rats markedly increased hepatocyte proliferation.

Source of increased HGF during liver regeneration. HGF is reported to increase in LSECs after liver injury (2). To determine whether this was due to increased expression of HGF in resident LSECs or to the influx of growth factor-rich BM-derived SPCs, PHx was performed in wild-type Lewis rats transplanted with Lew$\mathrm{Tg}$ (CAG-EGFP)ys BM. On day 3 after PHx, LSECs were isolated, sorted into $\mathrm{GFP}^{+}$and $\mathrm{GFP}^{-}$fractions, and examined for HGF gene and protein expression. Real-time PCR showed markedly higher $\mathrm{Hg} f$ gene expression in $\mathrm{GFP}^{+}$cells present in the elutriated to repopulation of LSECs after liver injury and their respective roles in liver regeneration. PHx led to a robust BM SPC progenitor cell response, defined here as proliferation of SPCs in the BM, mobilization of BM SPCs to the circulation, engraftment of SPCs in the liver, and differentiation to fenestrated LSECs. Three days after PHx, 25\% of LSECs were BM derived. After 2 weeks, the originally nonfenestrated, BM-derived SPCs had developed fenestrae grouped into sieve plates, the hallmark of differentiated LSECs.

Previous studies suggested that LSEC HGF expression increases after liver injury and that this increased HGF expression in LSECs promotes liver regeneration $(1,2)$. This study refines the concept by demonstrating that most of the increase in HGF does not come from the LSECs but is derived from an influx of HGF-rich BM SPCs that engraft after liver injury and that are present in the isolated LSEC fraction. This influx of BM SPCs promotes hepatocyte proliferation. Of note, hepatic stellate cells are a rich source of HGF, and contamination by these 




\section{Figure 5}

Engraftment of BM SPCs in the liver. Liver from wild-type rats, transplanted with BM from Lew-Tg(CAG-EGFP)ys rats, on day 5 after PHx. (A) GFP+ BM cells engraft along the sinusoid. (B) CD31 staining indicates LSECs. (C) The merged image of $\mathbf{A}$ and B demonstrates that GFP+ BM cells engraft as LSECs. Original magnification, $\times 100$. cells could confound the results. The method used to isolate LSECs, however, provides a population that is 99\% pure LSEC, as determined by uptake of formaldehyde-treated serum albumin. Thus, SPCs isolated from the LSEC fraction were not contaminated by stellate cells, and contamination cannot account for the high HGF expression.

BM SPCs were necessary for normal liver regeneration: BM suppression impaired liver regeneration, whereas infusion of SPCs promoted hepatocyte proliferation and restored normal liver regeneration. There was no difference in the effect of SPC infusion compared with BM infusion on hepatocyte proliferation and liver regeneration, indicating that SPCs are sufficient to account for the whole benefit of BM infusion. Although the infusion of SPCs on day 1 after PHx restored normal liver regeneration, infusion of SPCs on day 3 did not. This temporal divergence in the effect of SPC infusion is pertinent, because, in the rat PHx model, the paracrine effect of HGF released by nonparenchymal cells is thought to stimulate the second round of proliferation of hepatocytes, which occurs on day 2 in the rat (15). Furthermore, over $40 \%$ of intrahepatic SPCs are still proliferating on day 3 after PHx, and this likely reflects the need to produce additional LSECs to line the microcirculation of the regenerating liver.

The current study has identified and examined resident LSEC LRCs and progenitor cells. The label-retaining assay identifies putative stem cells (16-20), but demonstration that an LRC is a functional stem cell requires evidence of self-renewal, lineagespecific differentiation, and serial repopulation. LSEC LRCs in the liver are relatively numerous: 1 in 1,000 LSECs are resident LRCs. By comparison, slightly fewer than 1 in 10,000 BM mononuclear cells are stem cells (21). The resident SPC is identified by the presence of $\mathrm{CD} 133^{+}$, a classic progenitor cell marker, but it also has fenestrae organized in sieve plates, which shows that it is differentiating into an LSEC. On day 3 after PHx, 57\% of all SPCs within the liver were resident progenitor cells, but the BM-derived SPCs are the major source of HGF and have much higher proliferation rates. Thus, although our studies show that resident SPCs that have been isolated and infused into the liver after PHx can promote hepatocyte proliferation and liver regeneration, resident SPCs in their niche play a less important role in liver regeneration than BM SPCs.

The current study has 2 clinical implications. First, the observation that BM suppression impairs liver regeneration is an unexpected finding that may shed light on worsening liver disease and acute liver failure in BM-suppressed patients. Lack of compensatory liver regeneration may contribute to the $12 \%$ incidence of acute hepatic failure and death in patients with hepatitis B after hematopoietic cell transplantation (22) and to the high case-fatality rate of sinusoidal obstruction syndrome after myeloablative conditioning for hematopoietic cell transplantation (23). The finding that infusion of SPCs can overcome the effects of BM suppression provides new avenues for potential therapies for liver disease in BM-suppressed patients. Second, liver regeneration is impaired in chronic liver disease. The finding that recruitment of SPCs is necessary for liver regeneration raises questions about SPC recruitment in chronic liver disease.

In summary, this study has identified 2 sources of LSEC progenitors: within the liver, there are resident LSEC LRCs and fenestrated SPCs and, within the BM, there are nonfenestrated SPCs that substantially repopulate the sinusoids after liver injury and differentiate into fenestrated LSECs. The BM-derived SPC does not contribute significantly to normal turnover of LSECs or resident SPCs in adult rats, but BM SPCs are the major contributor to recovery from liver injury. Perhaps more importantly, the influx of BM SPCs provides most of the increase in LSEC HGF after injury and promotes hepatocyte proliferation and normal liver regeneration. When present in their niche, resident LSEC LRCs and SPCs play a lesser role in LSEC repopulation after liver injury than BM SPCs; however, when resident SPCs are isolated from the liver and infused after PHx, there is marked expansion of the SPCs with persistent engraftment as LSECs and SPCs.
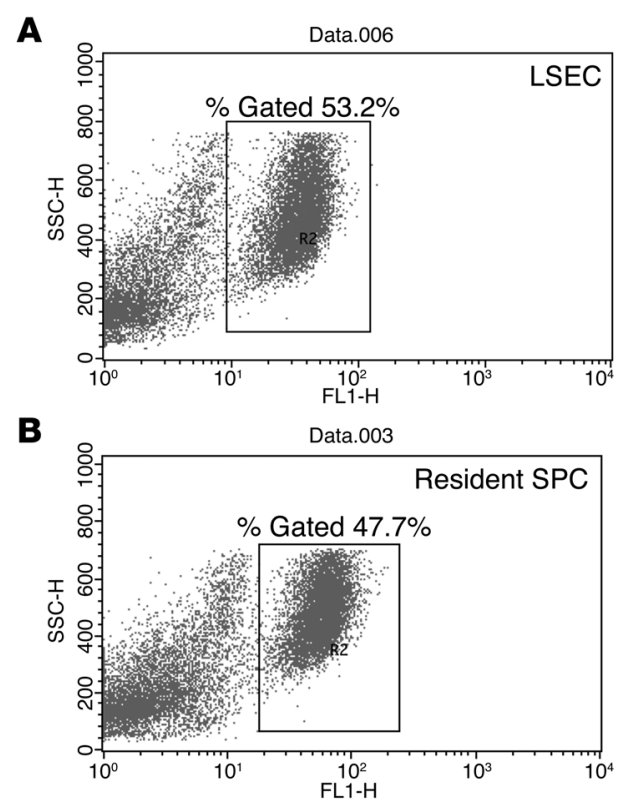

\section{Figure 6}

Persistence of engraftment and expansion. Resident SPCs were isolated from GFP+ transgenic rats, and $1 \times 10^{6}$ resident SPCs were infused into the tail veins of wild-type Lewis rats 3 days after $\mathrm{PHx}(n=3)$. Two months later, LSECs and resident SPCs were isolated and examined by FACS for GFP expression (shown on the $x$ axis). (A) The gated area shows $53.2 \%$ of LSECs are GFP+, i.e., BM derived. (B) The gated area shows $47.7 \%$ of resident SPCs are GFP+, i.e., BM derived. SSC-H, side scatter height; FL1-H, fluorescence intensity. 

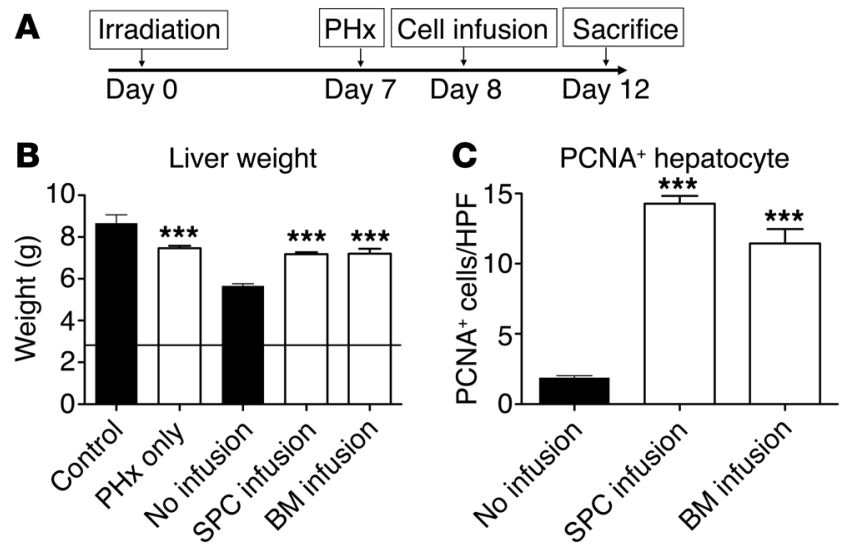

\section{Methods}

Materials. Chemicals were obtained from Sigma-Aldrich unless stated otherwise. The following antibodies were used: mouse anti-rat CD31 (Abcam); TRITC-conjugated anti-PCNA, rabbit anti-rat CD31, goat anti-Flt1, rabbit anti-Flk1, mouse anti-rat HGF, TRITC-conjugated donkey anti-rabbit IgG, and FITC-conjugated donkey anti-rabbit IgG (Santa Cruz Biotechnology Inc.); FITC-conjugated mouse anti-rat CD45, mouse anti-rat CD45, Alexa Fluor 488-conjugated mouse anti-rat BrdU, and PE-conjugated goat anti-mouse IgG (BD Biosciences); and Alexa Fluor 405-conjugated goat anti-mouse IgG and Alexa Fluor 405-conjugated goat anti-rabbit IgG (Invitrogen). Microbeads from the following kits were used: CD133 Cell Isolation Kit and Anti-FITC MultiSort Kit (Miltenyi Biotec).

Animals. Sprague-Dawley, Lewis, and Fischer rats were obtained from Harlan Corp. Breeding pairs of Lew- $\mathrm{Tg}(\mathrm{CAG}-\mathrm{EGFP}) \mathrm{ys}$ Lewis rats were obtained from the NIH Rat Resource and Research Center, University of Missouri.

PHx was performed under general anesthesia. $70 \%$ of liver was resected. Sham surgery consisted of laparotomy and gentle mobilization of the liver.

This study followed the guidelines outlined in the "Guide for the Care and Use of Laboratory Animals," prepared by the National Academy of Sciences and published by the NIH (NIH publication no. 86-23, revised 1985).

LSEC isolation. LSECs were isolated by collagenase perfusion, iodixanol density gradient centrifugation, and centrifugal elutriation as previously described $(12,13)$. Yields averaged 84 million cells per normal rat liver, with more than $95 \%$ viability. Purity of these cells is $99 \%$, as determined by uptake of formaldehyde-treated serum albumin (gift from BÅrd Smedsrod, University of Trømso, Trømso, Norway), a function specific to LSECs (24-26), and cells isolated by this protocol in our laboratory have fenestrae organized in sieve plates. Fewer than $1 \%$ of cells were Kupffer cells, as demonstrated by a peroxidase stain. Periportal and centrilobular LSECs were isolated as previously published (14).

SPC isolation. BM and peripheral blood SPCs were isolated by immunomagnetic selection for CD133 and CD45, followed by FACS sorting for CD $31^{+}$ cells for studies that required PCNA staining or CD133 immunomagnetic selection, followed by FACS sorting for CD45 and CD31 for other experiments. For double-label immunomagnetic selection, BM and peripheral blood mononuclear cells were incubated with anti-CD45 FITC antibody ( $1: 10$ dilution, 30 minutes at $4^{\circ} \mathrm{C}$ ) and incubated with anti-FITC microbeads ( $20 \mu \mathrm{l}$ beads for up to $10^{7} \mathrm{cells}$ ) for 30 minutes at $4^{\circ} \mathrm{C}$. After magnetic selection using the autoMACS Pro (Miltenyi Biotec), release reagent was used to clip off the magnetic bead. $\mathrm{CD} 45^{+}$cells were incubated with anti-CD133 microbeads ( $100 \mu$ l beads for up to $10^{8}$ cells) for 30 minutes at $4{ }^{\circ} \mathrm{C}$.

To obtain resident SPCs, a nonparenchymal fraction was obtained by collagenase perfusion and iodixanol density gradient centrifugation, as per LSEC isolation protocol (12). Elutriation fractions from the first elutriation

\section{Figure 7}

Infusion of SPCs restored normal liver regeneration in BM-suppressed rats. (A) Cartoon of the experimental design. (B) Liver weight was examined in control rats and in rats 5 days after $\mathrm{PHx}$ (day 12) in the following groups: nonirradiated rats ( $\mathrm{PHx}$ only), irradiated rats without cell infusion (no infusion), irradiated rats rescued with infusion of SPCs ("SPC infusion"), and irradiated rats rescued with BM cell infusion ("BM infusion") $(n=4)$. The horizontal line at 2.8 grams indicates the estimated liver weight immediately after two-thirds PHx. (C) Hepatocyte proliferation on day 5 after $\mathrm{PHx}$ was determined by PCNA immunostaining $(n=3) .{ }^{* \star *} P<0.001$ compared with no infusion group.

step were examined for $\mathrm{CD} 133^{+}$cells. Virtually all CD133+ cells were recovered from the same elutriation fraction as LSEC during the first elutriation step, and $\mathrm{CD} 133^{+}$cells isolated by this method were confirmed to be LSEC progenitors (see Results). Thus, resident SPCs were obtained by isolation of LSECs and immunomagnetic selection of the $\mathrm{CD} 133^{+}$fraction.

Immunostaining. Frozen sections of liver tissue were fixed with acetone, and coverslips with LSECs were fixed with $4 \%$ paraformaldehyde. Slides were incubated with anti-PCNA, anti-VEGF receptor 1, anti-VEGF receptor 2, anti-CD31, anti-CD45, or anti-BrdU antibody (1:100 dilution) at $37^{\circ} \mathrm{C}$ for 1 hour and examined by confocal microscopy (Zeiss LSM 510, Carl Zeiss Microimaging Inc.).

Label-retaining assay. Day 3 newborn pups (Sprague-Dawley rats) were injected with 7 doses of $50 \mu \mathrm{g} / \mathrm{g}$ BrdU s.c. in saline, given twice daily over 3.5 days. After 60 days, LSECs were isolated; immunomagnetically selected for CD133; immunostained for CD31, CD45, and BrdU; and examined by confocal microscopy.

Flow cytometry. $0.5 \times 10^{6}$ LSECs were incubated with PE-conjugated antiCD31 antibody (1:100 dilution) or TRITC-conjugated anti-PCNA antibody (1:100 dilution) at $4^{\circ} \mathrm{C}$ for 30 minutes and examined using a FACSCalibur (BD Biosciences). Data were analyzed using Cell Quest Pro software.


\section{Figure 8}

Role of BM-derived SPCs in liver regeneration. LSECs were isolated on day 3 after $\mathrm{PHx}$ from wild-type rats that had been transplanted with $\mathrm{BM}$ from Lew-Tg(CAG-EGFP)ys rats. (A) Hgf mRNA expression was measured by real-time PCR in GFP+ (BM-derived) and GFP- LSECs. $n=3$; ${ }^{* * *} P<0.001$. (B) HGF protein expression was determined by immunoblot and (C) quantified by densitometry in control LSECs (isolated from nonoperated rats) and in $\mathrm{GFP}^{+}$and GFP- LSECs isolated on day 3 after $\mathrm{PHx}(n=3) .{ }^{*} P<0.05,{ }^{*} P<0.01$ compared with GFP- LSECs after $\mathrm{PHx}$. (D) CD133+ cells from the LSEC fraction were immunostained for PCNA and sorted by FACS for GFP. $n=3$; ${ }^{* \star *} P<0.0001$ comparison of the percentage $\mathrm{PCNA}^{+}$cells in the GFP+ ${ }^{+}$and GFP- fractions. 
FISH. Freshly isolated LSECs were applied to slides by cytospin preparation at $30 \mathrm{~g}$ for 5 minutes. FISH was performed as previously published (8). Y chromosome was detected with FITC-labeled anti-digoxigenin (Boehringer Mannheim, Roche) and anti-fade mounting medium (Dako Corporation) containing $100 \mathrm{ng} / \mathrm{ml}$ propidium iodide (Molecular Probes). Cells were considered positive if a red nucleus with a single green dot was seen using a Zeiss LSM 510 confocal microscope. The presence of Y chromosome was determined in 20 cells per field in 15 random fields. The $\mathrm{Y}$ chromosome is only visualized when it is in the plane of visualization. To correct for nonvisualization of Y chromosome, LSECs were isolated from a control male rat, and the percentage of Y chromosome-positive cells was determined.

Irradiation. The lateral pelvis and hind limbs, with exclusion of the paws, were irradiated with $800 \mathrm{cGy}$ to 2 -cm depth using the $6 \mathrm{MeV}$ X-ray beam of a Varian 2300 c/d linear accelerator, located at the University of Southern California Norris Cancer Research Institute.

$B M$ transplantation. Cells were obtained from the BM of one tibia and femur from the donor. Recipients underwent $1,000 \mathrm{cGy}$ total body irradiation and were injected via tail veins with $50 \times 10^{6} \mathrm{BM}$ cells. BM was allowed to engraft for 2 to 3 months before use. Two BM transplantation models were used. BM from male Fischer rats was transplanted into female Fischer rats and tracked by FISH detection of the Y chromosome (8). BM from male Lew- $\operatorname{Tg}(\mathrm{CAG}-\mathrm{EGFP}) \mathrm{ys}$ Lewis rats was transplanted into wild-type female Lewis rats and tracked by GFP expression.

Statistics. Numerical data represent mean \pm SEM from at least 3 separate experiments. Values were compared by 2 -factor ANOVA with replication or 2-sided, 2-tailed Student's $t$ test, using Microsoft Excel (Microsoft) Analysis Toolpak. If ANOVA was significant, a posteriori comparison of individual points was performed using least significant difference. $P<0.05$ was considered significant.

1. Ding BS, et al. Inductive angiocrine signals from sinusoidal endothelium are required for liver regeneration. Nature. 2010;468(7321):310-315.

2. Maher JJ. Cell-specific expression of hepatocyte growth factor in liver: Upregulation in sinusoidal endothelial cells after carbon tetrachloride. J Clin Invest. 1993;91(5):2244-2252.

3. LeCouter J, et al. Angiogenesis-independent endothelial protection of liver: role of VEGFR-1. Science. 2003;299(5608):890-893.

4. Greene AK, et al. Endothelial-directed hepatic regeneration after partial hepatectomy. Ann Surg. 2003;237(4):530-535.

5. Nanji AA, Tahan SR, Wei Y, Sadrzadeh SM. Hepatic sinusoidal endothelial cell G1/S arrest correlates with severity of alcoholic liver injury in the rat. Gastroenterology. 1994;107(3):818-823.

6. Taniguchi E, Sakisaka S, Matsuo K, Tanikawa K, Sata M. Expression and role of vascular endothelial growth factor in liver regeneration after partial hepatectomy in rats. J Histochem Cytochem. 2001; 49(1):121-130.

7. Urbich C, et al. Soluble factors released by endothelial progenitor cells promote migration of endothelial cells and cardiac resident progenitor cells. J Mol Cell Cardiol. 2005;39(5):733-742.

8. Harb R, et al. Bone marrow progenitor cells repair rat hepatic sinusoidal endothelial cells after liver injury. Gastroenterology. 2009;137(2):704-712.

9. Xie G, Wang L, Wang X, Wang L, DeLeve LD. Isolation of periportal, mid-lobular and centrilobular rat liver sinusoidal endothelial cells enables study of zonated drug toxicity. Am J Physiol Gastrointest

Study approval. All protocols were reviewed and approved by the Institutional Animal Care and Use Committee at the University of Southern California to ensure ethical and humane treatment of the animals.

\section{Acknowledgments}

The authors thank Michelle MacVeigh-Aloni for her assistance with immunostaining and confocal microscopy and Thomas Kampp for his kind assistance with the irradiation. This work was supported by NIH grant R01-DK46357 (L.D. DeLeve) and by the Histology and Microscopy sub-core of the University of Southern California Research Center for Liver Diseases (NIH grant P30DK048522).

Received for publication April 29, 2011, and accepted in revised form January 25, 2012.

Address correspondence to: Laurie D. DeLeve, University of Southern California, Keck School of Medicine, Division of Gastrointestinal and Liver Diseases, 2011 Zonal Avenue, HMR 603, Los Angeles, California 90033, USA. Phone: 323.442.3248; Fax: 323.442.3238; E-mail: deleve@usc.edu.

Lin Wang's present address is: Department of Hepatobiliary Surgery, Xijing Hospital, Fourth Military Medical University, Xi'an, China.

Guanhua Xie's present address is: Division of Gastroenterology, Department of Medicine, Duke University Medical Center, Durham, North Carolina, USA.

Liver Physiol. 2010;299(5):G1204-G1210.

10. Torsney E, Xu Q. Resident vascular progenitor cells. J Mol Cell Cardiol. 2011;50(2):304-311.

11. Psaltis P, Harbuzariu A, Delacroix S, Holroyd E, Simari R. Resident vascular progenitor cells--diverse origins, phenotype, and function. J Cardiovasc Transl Res. 2011;4(2):161-176.

12. DeLeve LD, Wang X, McCuskey MK, McCuskey RS. Rat liver endothelial cells isolated by antiCD31 immunomagnetic sorting lack fenestrae and sieve plates. Am J Physiol Gastrointest Liver Physiol. 2006;291(6):G1187-G1189.

13. Steffan AM, Gendrault JL, McCuskey RS, McCuskey PA, Kirn A. Phagocytosis, an unrecognized property of murine endothelial liver cells. Hepatology. 1986;6(5):830-836.

14. Xie G, Wang L, Wang X, DeLeve LD. Isolation of periportal, midlobular, and centrilobular rat liver sinusoidal endothelial cells enables study of zonated drug toxicity. Am J Physiol Gastrointest Liver Physiol. 2010;299(5):G1204-G1210.

15. Michalopoulos GK. Liver regeneration after partial hepatectomy: critical analysis of mechanistic dilemmas. Am J Pathol. 2010;176(1):2-13.

16. Tsujimura A, et al. Proximal location of mouse prostate epithelial stem cells: a model of prostatic homeostasis. J Cell Biol. 2002;157(7):1257-1265.

17. Oliver JA, Maarouf O, Cheema FH, Martens TP, AlAwqati $Q$. The renal papilla is a niche for adult kidney stem cells. J Clin Invest. 2004;114(6):795-804.

18. Potten CS, Morris RJ. Epithelial stem cells in vivo. J Cell Sci Suppl. 1988;10:45-62.

19. Morris RJ, Potten CS. Slowly cycling (label-retain- ing) epidermal cells behave like clonogenic stem cells in vitro. Cell Prolif. 1994;27(5):279-289.

20. Kuwahara R, Kofman AV, Landis CS, Swenson ES, Barendswaard E, Theise ND. The hepatic stem cell niche: identification by label-retaining cell assay. Hepatology. 2008;47(6):1994-2002.

21. Kiehl MJ, Yilmaz OH, Terhorst C, Morrison SJ. SLAM family receptors distinguish stem and progenitor cells and reveal endothelial niches for stem cells. Cell. 2005;121(7):1109-1121.

22. Lau GKK, Strasser SI, McDonald GB. Hepatitis virus infections in patients with cancer. In: Wingard JR, Bowden RA, eds. Management Of Infection In Oncology Patients. London, United Kingdom: Martin Dunitz Publishing; 2003:321-342.

23. Sakai M, Strasser SI, Shulman HM, McDonald SJ, Schoch HG, McDonald GB. Severe hepatocellular injury after hematopoietic cell transplant: incidence, etiology and outcome. Bone marrow Transplant. 2009;44(7):441-447.

24. Blomhoff R, Eskild W, Berg T. Endocytosis of formaldehyde-treated serum albumin via scavenger pathway in liver endothelial cells. Biochem J. 1984;218(1):81-86.

25. Blomhoff R, Smedsrød B, Eskild W, Granum PE, Berg T. Preparation of isolated liver endothelial cells and Kupffer cells in high yield by means of an enterotoxin. Exp Cell Res. 1984;150(1):194-204.

26. Eskild W, Kindberg GM, Smedsrod B, Blomhoff $\mathrm{R}$, Norum KR, Berg T. Intracellular transport of formaldehyde-treated serum albumin in liver endothelial cells after uptake via scavenger receptors. Biochem J. 1989;258(2):511-520. 